\title{
Raman, Hyper-Raman, Hyper-Rayleigh and Two-Photon Excited Luminescence Microspectroscopy in an Optical Tweezers System.
}

\author{
A. Fontes*, K. Ajito**, A. M. De Paula***, A. R. Neves*, W. L. Moreira*, L. C. Barbosa* and C. L. \\ Cesar* \\ * Instituto de Física Gleb Wataghin - Universidade Estadual de Campinas 13083-970 - Campinas - \\ SP - Brazil. \\ ** NTT Basic Research Laboratories - Nippon Telegraph and Telephone (NTT) \\ Corporation 243-0198 - Kanagawa - Japan. \\ *** Universidade São Francisco - Laboratório de Nano-Espectroscopia Óptica 12916-900 - \\ Bragança Paulista - SP - Brazil.
}

Single beam optical tweezers have been used as a tool to manipulate biological material at cellular level, as well as to measure mechanical properties such as forces at femtonewton scale and stiffness or elasticity of membranes and single DNA macromolecule [1-3]. We used the optical tweezers to study disease related to the mechanical properties of individual red blood cells [4] and we demonstrated the importance of using spectroscopic techniques while manipulating particles and living cells. The ability of performing spectroscopy in a living microorganism optically trapped in any desired neighborhood means that we could dynamically observe the chemical reactions and/or mechanical properties change in real time. Therefore, we decided to set up an Optical Tweezers plus a Raman system like the one described in reference [5,6]. We present a homemade set-up confocal spectrometer using a Spectra Pro $300 \mathrm{i}$ - Acton Research Corporation $30 \mathrm{~cm}$ triple grating monochromator equipped with a Princeton Instruments liquid cooled back illuminated CCD using a femtosecond Ti:sapphire laser, Spectra Physics Tsunami. Previous works used only cw lasers. In our system we have the possibility of using the Tsunami and/or a cw Ti:sapphire laser, Spectra Physics model 3900S. The use of a femtosecond laser with or without a cw laser opens up a great number of different applications and spectroscopies, as discussed below. The drawback of a femtosecond laser is the intrinsic broad linewidth associated with time-frequency Fourier transform $\Delta v \Delta \tau \geq 1 / 2$. If the issue is a great spectral resolution we can use the narrow cw laser line or narrow down the femtosecond laserline with an intracavity slit. It is also possible to narrow the femtosecond laser line with an external band pass filter but loosing the power out of the filter range. With the cw laser we used two supernotch filter to reject the laser line from the monochromator. The linewidth of a 100 femtosecond pulse, however, is larger than the $350 \mathrm{~cm}^{-1}$ of the supernotch filter, which leads to a leakage of the laser line out of the supernotch filter spectral range. Nonetheless, because the leaking power was low, it was still possible to observe the Raman lines superimposed to the tail of the Gaussian laser line leakage. Only using the band pass filter it was possible to avoid this leakage. Figure 1 shows the obtained Raman spectra for a trapped polystyrene sphere, a red blood cell and a $\mathrm{ZnSe}$ sample. One possible application for the pulsed laser is the excitation of the luminescence by two photon absorption (TPA). Two photon optical processes only happen when two photons meet at the same time and at the same spot, which happens much more frequently for a pulsed laser (photons at the same time) and at the laser focus (photons at the same spot). The TPA allow the simultaneously observation of the Raman, at the infrared region, and the luminescence, at the visible region. Figure 2 shows the TPA excited luminescence of $\mathrm{ZnSe}$. The systems that use the absorption of two photons or more are confocal by itself, because at the right pump power, the signal is generated only at the spot size of the exciting laser. This makes the signal collection optics not so 
important as in the conventional confocal techniques [7]. Some of the advantages of using a infrared laser for two photon absorption in living cells are the resolution and the absence of damage caused by the heating. The drawback of luminescence for living cells is that the nonradiative decaying usually requires cell staining that can kill them. Fortunately, not only luminescence can be excited by the femtosecond laser, but Hyper-Raman and Hyper-Rayleigh as well. In the classical model the polarization is given by $\vec{P}(\vec{E})=\vec{P}_{o}+\chi^{(1)}\left(q_{j}\right): \vec{E}+\chi^{(2)}\left(q_{j}\right): \vec{E} \vec{E}+\ldots$. The Taylor expansion of the $\chi^{(1)}$ gives the Rayleigh/Raman scattering, while the second order susceptibility Taylor expansion $\chi^{(2)}=\chi^{(2)}(0)+\left(\partial \chi^{(2)}(0) / \partial q_{j}\right) q_{j \pm} e^{ \pm i \omega_{j} t}$ gives the Hyper-Rayleigh at $2 \omega$ and the Stokes/Anti-Stokes Hyper-Raman at $2 \omega \pm \omega_{j}$ frequencies. The Hyper-Rayleigh and Hyper-Raman tensors are different and, in most cases, complementary to the Raman and Rayleigh ones. Only the huge intensity of a pulsed laser allows one to access the second order tensors. We have tested the ability of our system to observe the Hyper-Rayleigh and Hyper-Raman with a ZnSe sample as shown in figure 2. Finally, there is also the possibility to observe coherent Raman effects using two laser lines (the pulsed and the cw Ti:sapphire) at different wavelengths such as Coherent Anti-Stokes Raman Spectroscopy (CARS). Although the CARS information's refer to the same Raman tensor of the conventional measurement, it allows, due to the much more strong signal, taking the measurements much faster, which can be critical for scanned images and biological samples [8]. These spectroscopic techniques and the optical tweezers mounted in only one system can provide a thorough analysis of all kinds of living cells (flagellate or not) and other single trapped particles.

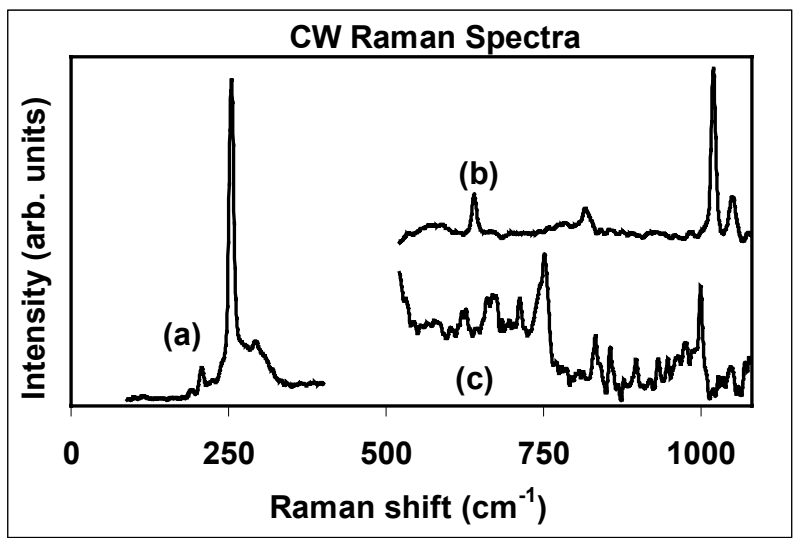

Fig. 1. Raman lines of a single polystyrene microsphere (b) $(2 \mathrm{~s}, \sim 20 \mathrm{~mW})$ and red blood cell (c) $(2 \mathrm{~min}, \sim 20 \mathrm{~mW})$ captured by optical tweezers and of $\mathrm{ZnSe}$ (a) $(1 \mathrm{~s}, \sim 35 \mathrm{~mW})$. CW wavelength in $785 \mathrm{~nm}$.

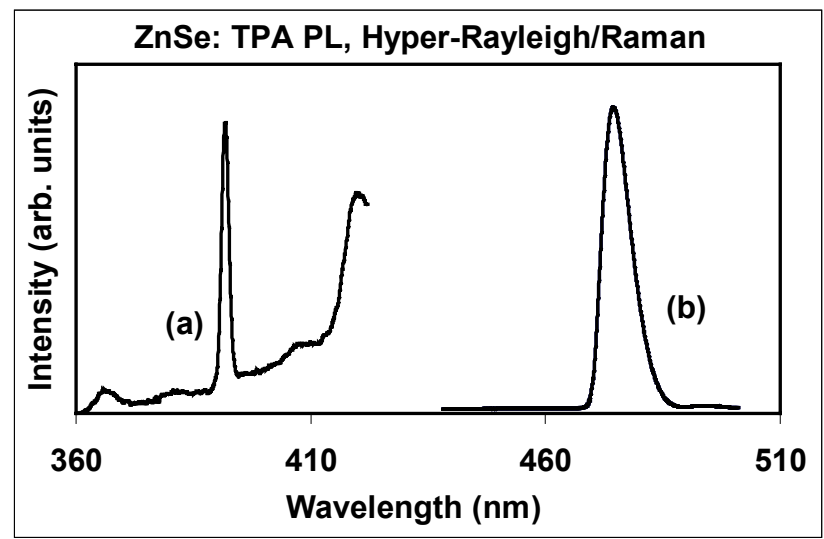

Fig. 2. (a) Hyper-Rayleigh (center of the figure), Stokes ( 420nm) and Anti-Stokes Hyper-Raman (50s, $\sim 200 \mathrm{~mW}$ average power) and (b) TPA fluorescence of $\mathrm{ZnSe}(1 \mathrm{~s})$.

[1]. A. Ashkin, J.M. Dziedzic, Science, 235 (1987) 1517.

[2]. K. Sakata-Sogawa et al., Eur. Biophys. J. 27 (1998) 55.

[3]. K. Konig et al., Cell. Mol. Biol. 42 (1996) 501.

[4]. ML. Barjas-Castro et al, Transfusion 429 (2002) 1196.

[5]. K. Ajito, K. Torimitsu, Applied Spectroscopy 564 (2002) 541.

[6]. C. Xie et al, Optics Letters 274 (2002) 249.

[7]. K. Konig, Hist. Cell. Biol. 114 (2000) 79.

[8]. A. Zumbusch et al, Physical Review Letters 8220 (1999) 4142. 